\section{Anatomy for beginners}

\section{http://www.channel4.com/science/microsites/A/anatomy/}

This site accompanies the controversial television series Anatomy for Beginners. Only a simple overview of functional anatomy is provided covering movement, circulation, digestion and reproduction. Numerous photographs from public anatomy dissections are included. This is clearly aimed at a non-medical audience; therefore, the scientific benefit is limited.*

\section{BBC The Human Body}

\section{http://www.bbc.co.uk/science/humanbody/body/}

This is a useful interactive site aimed at the general public. The main systems are briefly covered with helpful diagrams, interactive zones and useful facts and figures. This may be a good resource for patients, but the lack of detail limits its benefit for surgeons. **

\section{Breaking bad news}

H ZAIDI

Bradford Royal Infirmary, Bradford, West Yorkshire, UK

\section{CORRESPONDENCE TO}

Dr Hammad Zaidi, Clinical Fellow Surgery, Bradford Royal Infirmary, Duckworth Lane, Bradford BD9 6RJ, UK

E: drhammadraza@yahoo.com

Breaking bad news is one of a physician's most difficult duties; yet medical education typically offers little formal preparation for this daunting task. One source ${ }^{1}$ defines bad news as 'any news that drastically and negatively alters the patient's view of her or his future'. Without proper training, the discomfort and uncertainty associated with breaking bad news may lead physicians to disengage from patients emotionally. Numerous studies show that patients generally desire frank and empathetic disclosure of a terminal diagnosis or other bad news. Following is a review of selected internet sites which provide insight to this topic and some up-to-date information and tips to breaking bad news.

\section{Reference}

1. Buckman R. Breaking bad news: why is it so difficult? BMJ 1984; 288 1597-9.

\section{www.aafp.org}

This web site was originally created by the American Association of Family Physicians. It is simple yet highly informative. It describes the basic steps involved in breaking a bad news. The use of ABCDE pneumonic makes the task very simple and explicable. This site is well constructed and a 'must read' for beginners.

\section{www. breakingbadnews.co.uk}

This website is designed to provide guidelines and practical suggestions to UK healthcare professionals on how to communicate any medical information effectively and compassionately, and respond to a patient's and family's feelings and needs. The site discusses guidelines, strategies and resources. It outlines strategies to handle various difficult scenarios faced routinely in clinical practice. A good site for rehearsal and practice. But it could have been excellent if upgraded by practice videos and interactive materials.

\section{www.communityoncology.net}

Community Oncology is a journal website which publishes articles related to clinical research in the community setting. The website has broad relevance to the problems faced by clinical practitioners. It is a reliable, up-to-date and relevant clinical site which gives guidance on how to tackle common problems encountered in clinical practice. It describes in detail the famous SPIKE strategy used for breaking bad news, which includes: S, setting; P, preparation; I, invitation; K, knowledge; and E, empathy. $A$ 'must read' site which eases the difficulties and removes any doubts regarding this difficult to tackle issue.

\section{www.skillcascade.com}

This website has been made possible with the support of Eastern Deanery and the resources are provided with the help of East Anglia communications skills cascade facilitators to promote and support the teaching of communication skills in health care. This web site covers important topics like preparation, setting the scene, sharing of information, being sensitive to a patient, planning and support, follow-up and closing. This site also gives information on various course plans, provides handouts, discusses specific issues and also gives good tips on communication skills.

\section{www.gptraining.net}

Welcome to gp-training.net, a new website derived from the Well Close Square website, one of the first GP surgeries in the UK to have its own web pages. The extensive site is divided into areas for doctor information and patient-related information. You can access the Neighbors 5 stage model of consultation used for breaking bad news. It is explained in a very simple yet logical manner. This is a well laid out site which is easy to read and follow.

\section{Thyroid disease}

\author{
T SINGH ${ }^{1}$, S RANDHAWA ${ }^{2}$, A NAWAZ
}

Departments of ${ }^{1}$ Ear, Nose and Throat, and ${ }^{2}$ Medicine (Gastroenterology), New Cross Hospital, Wolverhampton, UK

\section{http://www.endocrinesurgeon.co.uk/thyroid/index.html}

When searched for on Google, MSN or Askjeeves, information on thyroid disease is mainly provided by consultant endocrine or ENT surgeons. This website is a good example and was set up by two endocrine surgeons, a specialist endocrine anaesthetist and three biochemists. It certainly wins a prize for accessibility. This website is very easy to navigate. It goes through the basics of 
'what is the thyroid gland and its functions' and then by clicking on the icons, it takes one through different disease conditions and ways to manage thyroid problems, including the role of surgery. The only downside to this website is the excessive amount of medical jargon used. As it stands, it surely gives useful information for an overview on the subject. This site is a must visit for medical students and junior doctors.

\section{http://www.endocrineweb.com/thyroid.html}

This website is a well designed and a valuable source of information for medical students or junior doctors looking for facts on thyroid disease. It makes thyroid disease much easier to understand. There are many pictures, ultrasound scans and diagrams to make aid understanding of the pathophysiology, investigations and treatment of thyroid disease. Although it has useful links, navigation may be a problem as the links change every time one goes to a different web page. The website claims that it has over 100 pages of information on thyroid disease alone. Unlike many other websites, it stresses on the basics and goes into detail about different subheadings of thyroid disease. Surely worth a dabble for medical students and junior doctors.

\section{http://www.thyroidsurgery.org/}

This US-based Association of Thyroid Surgeons site emphasises the risks of thyroid surgery and how nerve monitoring technology can prevent injury. There is very little (sometimes no) literature on the anatomy, pathophysiology or management of thyroid disease. Keeping in mind that the website is managed by an association, it tells you where you can find a 'qualified thyroid surgeon'. Be aware, there are many sites like this offering various thyroid operations at their centres. This site is not very useful, except to patients who want to go privately for their thyroid disease in America. Not helpful for patients or any other medical professionals.

\section{http://cpmcnet.columbia.edu/dept/thyroid/index.html}

The New York Thyroid Center website was founded by Dr Paul LoGerfo who contributed much to thyroid and parathyroid surgery.
The site is very well designed, making it easy to use and navigate. It gives a very good overview of thyroid disease under various subheadings. There is very little medical jargon used; therefore, it is also useful for patients. On the downside, it contains only a few illustrations but the literature compensates for this deficiency. This website also has a postoperative instruction section concentrating on the incision, pain, problems with voice, thyroid replacement therapy, etc. Another good section on this website is on radioactive iodine, concentrating on the indications, advantages and disadvantages. It also touches on radionuclide scanning. A very useful website both for patients and surgeons.

http://www.endocrinologist.com/thyroid.htm\#Underactive\%20Thyroid The Diabetes \& Hormone Center of the Pacific site is aimed at patients. The contents are provided by Dr David Fitz-Patrick, who is also the director of this centre. The site is initially divided into the introduction, hyperthyroidism and hypothyroidism and thyroid nodules. The initial page shows a few cartoon illustrations of the thyroid gland in the neck and facial features of hyper- and hypothyroidism with a brief description of symptoms. Do not despair as these pictures have hyperlinks that are extremely good. These links will take you to pages of life pictures. Some of these may be disturbing to patients. The medical jargon used is minimal; therefore, it is useful to patients. Overall, an excellent patient-orientated website that is difficult to find fault with.

\section{http://www.thyroidsurgeon.org/index.html}

Although the David Scott-Coombes website is set up to promote private practice, one must give credit to $\mathrm{Mr}$ Scott-Coombes for a very neat and easy browse through site. The icons are arranged in a systematic order though it lacks pictures and diagrams. The information presented is in the form of questions and answers. Most of the frequently asked questions are answered here. Medical terms, like the course and function of the recurrent laryngeal nerve, are clearly explained to viewers in lay terms. This site may not be of much use to doctors, but is surely worth browsing by patients. 